\title{
SIMPLE MODELS OF PROTEINS WITH REPULSIVE NON-NATIVE CONTACTS
}

\author{
Mai Suan Li and Marek Cieplak \\ Institute of Physics, Polish Academy of Sciences, Al. Lotnikow 32/46, 02-668 Warsaw, Poland
}

\begin{abstract}
The Go model is extended to the case when the non-native contact energies may be either attractive or repulsive. The folding temperature is found to increase with the energy of non-native contacts. The repulsive non-native contact energies may lead to folding at $T=0$ for some two-dimensional sequences and to reduction in complexity of disconnectivity graphs for local energy minima.
\end{abstract}

PACS numbers: 71.28.+d, 71.27.+a

Functionally useful proteins are sequences of amino acids that fold rapidly under appropriate conditions (temperature range, acidity of the water solution etc) into their native states commonly assumed to be their ground state configurations [1]. The dynamics of folding is akin to motion in a rugged free energy landscape [2] and it crucially depends on two factors: the interactions between the amino acids and the target conformations. In this paper, we focus on the role of non-native contact energies in the folding process. We study this issue in a simple model which is an extension of the so called Go model proposed by Go and Abe [3]

Specifically, we consider the standard twodimensional lattice model of 16 monomers. Its Hamiltonian is as follows

$$
H=\sum_{i<j} \alpha_{i j} \Delta_{i j}
$$

where $\Delta_{i j}=1$ if monomers $i$ and $j$ are in contact and $\Delta_{i j}=0$ otherwise (monomers $i$ and $j$ are considered to be in contact if they are seperated by one lattice bond and $|i-j| \neq 1$ ). The quantity $\alpha_{i j}=-1$ if monomers $i$ and $j$ are in contact in the native conformation and $\alpha_{i j}=\alpha$ otherwise. The sequence is thus defined by the native conformation. We allow $\alpha$ to be attractive $(\alpha<0)$ or repulsive $(\alpha>0)$. In what follows $\alpha$ is assumed to be larger than -1.0 so that the native state is guaranteed to be a maximally compact conformation. In the original Go model [3] $\alpha=0$. It should be noted that using model (1) one can monitor the effect of non-native contact energies by varying only one parameter $\alpha$. Furthermore, the two-dimensional model is simple enough to study the effect of the target conformations on the folding dynamics.

It should be noted that there have already been several studies of models with repulsion in the nonnative contacts: a Gaussian model 仴, a designed model [5], and the so called HP+ model [6]. The generalized Go model that we study here allows one to vary the strength, and sign, of the non-native contacts relative to the native ones.

We focus on 4 target conformations shown in Fig. 1. There are 37 compact conformations (for the twodimensional 16-monomer chain one has 69 compact $4 \times 4$ conformations but for the Go-like model only 38 of them remain different due to the end-to-end reversal symmetry and one is not accessible kinetically) which may act as the native conformations. Among these $S_{1}$ and $S_{4}$ shown in Fig. 1 are the two fastest folders at $T \neq 0$, whereas $S_{2}$ and $S_{3}$, also shown in Fig. 1, have intermediate folding properties.
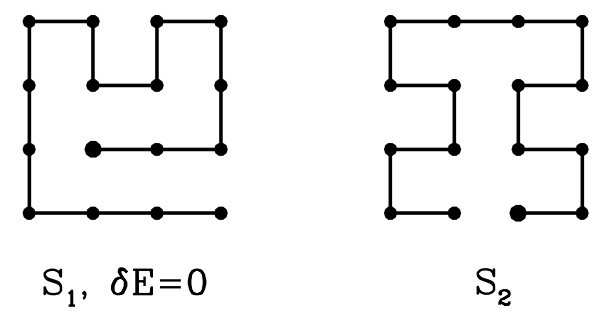

$\mathrm{S}_{2}$
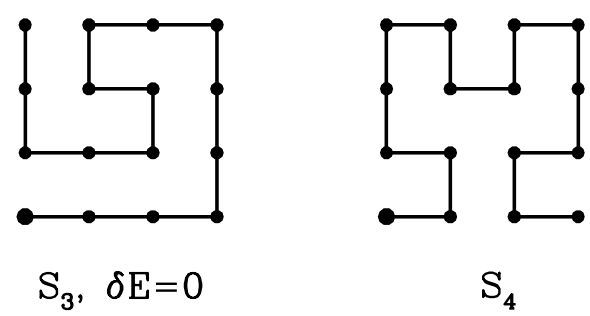

FIG. 1. Four sequences studied in this paper. $S_{1}$ and $S_{3}$ have $\delta E=0$.

It should be noted that the repulsive non-native contact interaction are expected to improve the 
foldicity 4 (6) because they restrict the size of the relevant phase space. In our case, however, this effect becomes so dramatic that for $\alpha>0.1$ sequences $S_{1}$ and $S_{3}$ can fold with a finite $t_{\text {fold }}$ even at $T=0$. Such an exotic phenomenon has been also observed in the HP model for some 13-monomer chains [7] so it is not restricted to the Go-like sequences that we study. Here, we study how this arises as a function of $\alpha$ and show how do folding characteristics, i.e. characteristic temperatures and folding times, depend on $\alpha$. The ability of $S_{1}$ and $S_{3}$ to fold at $T=0$ may be partly understood by the fact that for these sequences the repulsive non-native interactions reduce the number of local minima by two orders of magnitude compared to the case of $\alpha<0$. Furthermore, repulsive interactions dramatically affect partitioning of the phase space into regions associated with the local energy minima. We demonstrate this by using the disconnectivity graph technique [8] and show, in particular, that connectivities to the folding funnel become simplified significantly.

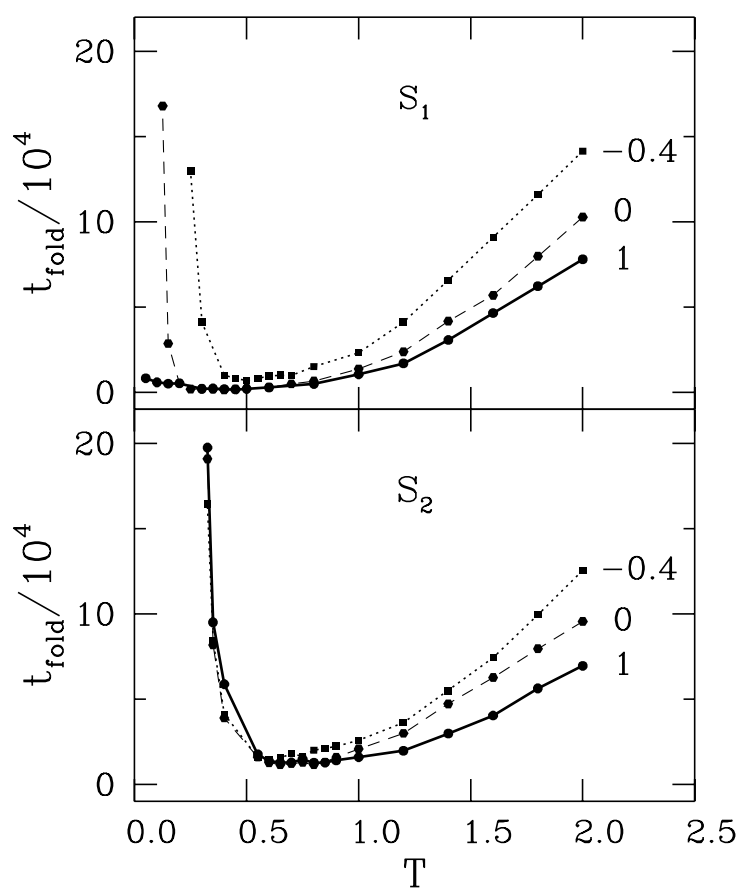

FIG. 2. Temperature dependence of $t_{f o l d}$ for $S_{1}$ and $S_{2}$ and for 3 selected values of $\alpha, \alpha=-0.4,0$ and 1 . The results are based on 2 - 4 batches, each containing 200 trajectories from initial random conformations.

We have found that the folding temperature, $T_{f}$, increases with $\alpha$. This result agrees with that of Camacho [9] for an effectively zero-dimensional model
[10]. We obtain it, however, not only by the numerical calculations for the lattice model but also by the analytical argument. The minimum folding time, $t_{\text {min }}$, defined at the temperature where the folding is fastest has found to decrease with $\alpha$ and it gets saturated for $\alpha \rightarrow \infty$.

The folding dynamics of a chain is studied by a Monte Carlo procedure that satisfies the detailed balance condition 11, and was motivated by the studies presented in Ref. [7, 12]. The dynamics allows for single and two-monomer (crankshaft) moves. For each conformation of the chain one has $A$ possible moves and the maximum value of $A, A_{\max }$, is equal to $A_{\max }=N+2$. In our 16-monomer case $A_{\max }=18$. For a conformation with $A$ possible moves, probability to attempt any move is taken to be $A / A_{\max }$ and probability not to do any attempt is $1-A / A_{\max }[7,12$. In addition, probability to do a single move is reduced by the factor of 0.2 and to do the double move - by 0.8 [12,7]. The attempts are rejected or accepted as in the standard Metropolis method. The folding time is equal to the total number of Monte Carlo attemps divided by $A_{\max }$.

We have carried out the Monte Carlo simulations to determine the dependence of the median folding time, $t_{f o l d}$, on $T$ and $\alpha$. The results for $S_{1}$ and $S_{2}$ are shown in Figure 2. For each temperature, $t_{\text {fold }}$ is obtained based on 200 independent runs starting from random configurations. The results are averaged over 2 - 4 batches, of 200 trajectories each.

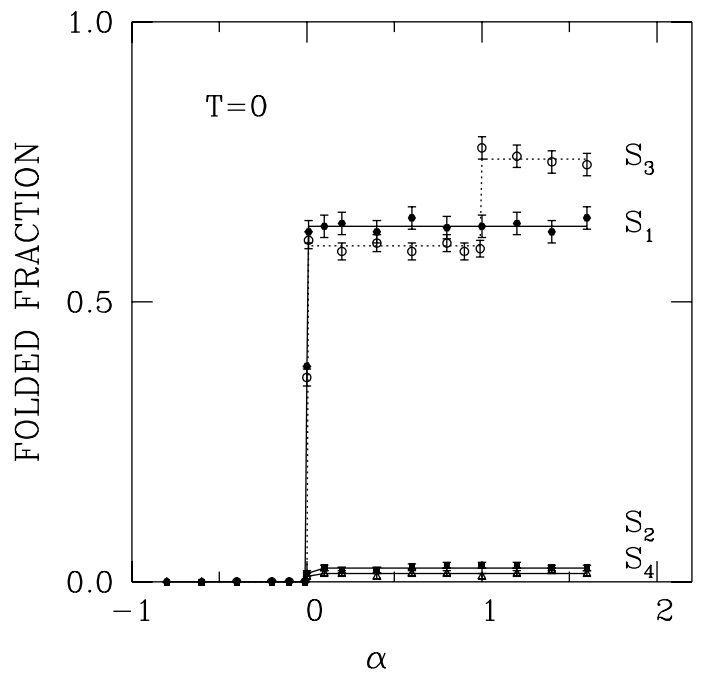

FIG. 3. Dependence of the folded fraction on $\alpha$ at $T=0$ for 4 sequences shown in Figure 1. The results are averaged over 4 - 6 simulations, each corresponding to 200 trajectories. 
For sequence $S_{2}$ the standard U-shape [13 for the temperature dependence of $t_{\text {fold }}$ is observed for all values of $\alpha$. In other words, no qualitative change occurs if the non-native contact energies change from attractive to repulsive. In the case of sequence $S_{1}$, however, for $\alpha=1$ the standard U-shape disappears suggesting that the glass transition temperature $T_{g}$ which is operationally defined as the value of the temperature at which the median time is equal to some cut-off value (usually this cut-off value is chosen to be 300000 Monte Carlo steps for the twodimensional 16-monomer chain [13]) becomes zero. In the standard scenario, at low temperatures the system may get trapped in some local minima and the folding process becomes extremly slow. The folding time is then governed by the Arrhenius law, $t_{\text {fold }} \sim \exp (\delta E / T)$ [14], where $\delta E$ is the energy barrier energy (at $T=0$ one has $t_{\text {fold }} \rightarrow \infty$ ). Thus, the absence of the $U$-shape dependence suggests that the energy barrier $\delta E=0$.

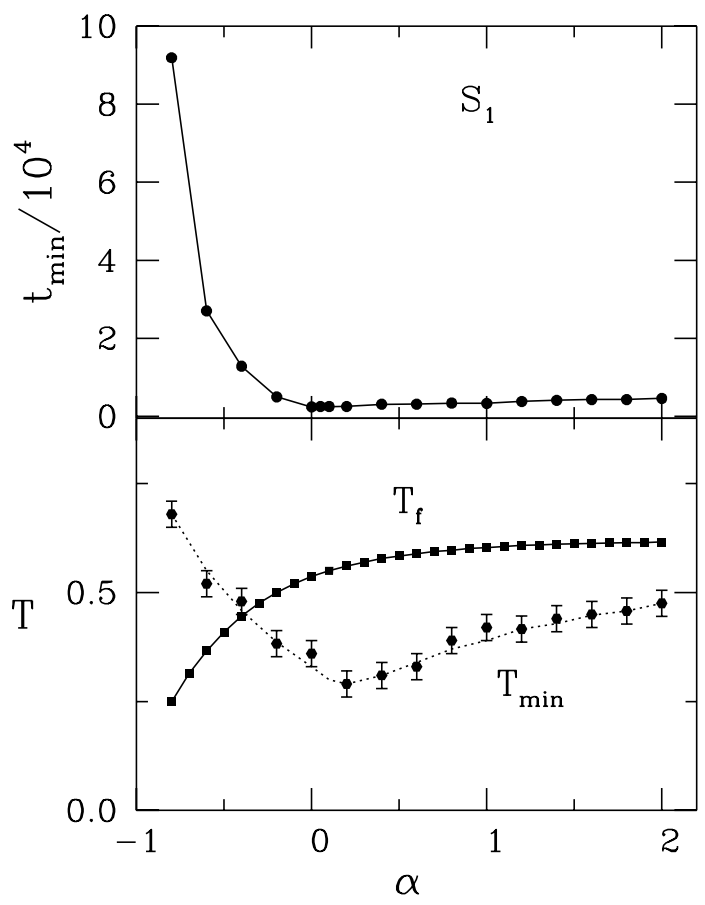

FIG. 4. Dependence of $T_{f}, T_{\min }$ and $t_{\min }$ for sequence $S_{1}$ on $\alpha$. The results are averaged over 4 batches, each containing 200 trajectories.

In order to know whether $\delta E$ is exactly zero or not one has to study the folding at $T=0$. At zero temperature the system gets trapped in some local minimum or in the native state. If the fraction of the trajectories from random conformations fold into the native state is bigger than $50 \%$, then the chain is said to be folded and $\delta E=0$.

The fraction of folded trajectories is shown in Fig. 3 for $S_{1}, S_{2}, S_{3}$ and $S_{4}$. For $S_{1}$ and $S_{3}$ this fraction becomes bigger than $50 \%$ for $\alpha>\alpha_{c}$, where $\alpha_{c} \approx$ 0.1 . Sequences $S_{2}$ and $S_{4}$ have $\delta E \neq 0$ for any value of $\alpha$. It is interesting to mention that $S_{4}$ folds even faster than $S_{1}$ at $T \neq 0$ but its foldicity becomes much worse at $T=0$. Furthermore, the folding rate of $S_{3}$ at $T \neq 0$ is slower than for the three other sequences and yet $S_{3}$ can fold at $T=0$. Thus the geometry of the native targets has a dramatic effect on the folding at $T=0$. Among the 37 maximally compact $4 \times 4$ structures it is only $S_{1}$ and $S_{3}$ that do not obey the Arrhenius law.

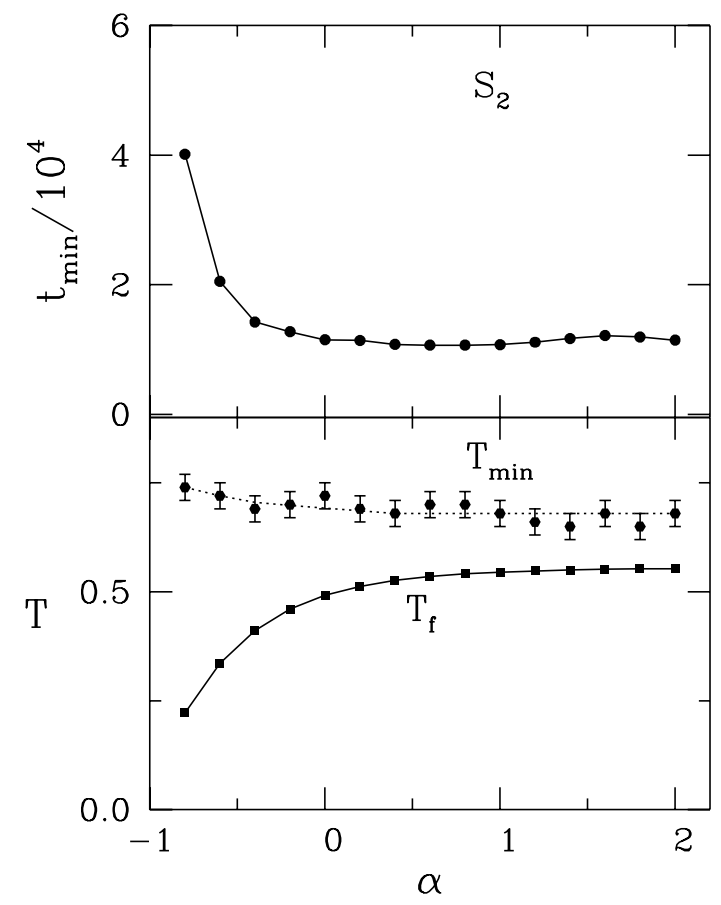

FIG. 5. The same as in Figure 4 but for sequence $S_{2}$.

We study the dependence of $T_{f}$ on $\alpha$ for two typical sequences $S_{1}$ and $S_{2}$ shown in Fig. 1. The total number of conformations of the 16-monomer chain is only 802075 [15,16] and is amenable to exact enumeration. This allows for an exact evaluation of the equilibrium parameters such as the folding temperature $T_{f}$. The latter is defined as a temperature at which the probability of occupancy of the native state is $1 / 2$. The results for sequences $S_{1}$ and $S_{2}$ are shown in Fig. 4 and 5 . Obviously, $T_{f}$ increases with $\alpha$ but this dependence gets weaker for larger values of $\alpha$. 
The increase of $T_{f}$ with $\alpha$ may be understood in the following simple way. Let $P_{\Gamma_{0}}(\alpha)$ be the probability of occupying the native state of conformation $\Gamma_{0}$. Then

$$
P_{\Gamma_{0}}(\alpha)=\frac{\exp ^{-\beta E_{\Gamma_{0}}}}{\sum_{\Gamma} \exp ^{-\beta \alpha n-\beta E_{\Gamma}^{\prime}}},
$$

where $E_{0}$ is the energy of the native state, $n$ is the number of non-native contacts and $E_{\Gamma}^{\prime}$ is the part of energy in conformation $\Gamma$ which corresponds to the native contacts. Then

$$
\frac{\partial P_{\Gamma_{0}}}{\partial \alpha} \geq \beta<n>_{T} \geq 0,
$$

where $\langle n\rangle_{T}$ is the average number of non-native bonds at temperature $T$. So the probability of being in the native state cannot decrease with $\alpha . T_{f}$, therefore, should increase with increasing $\alpha$ and then become $\alpha$-independent.

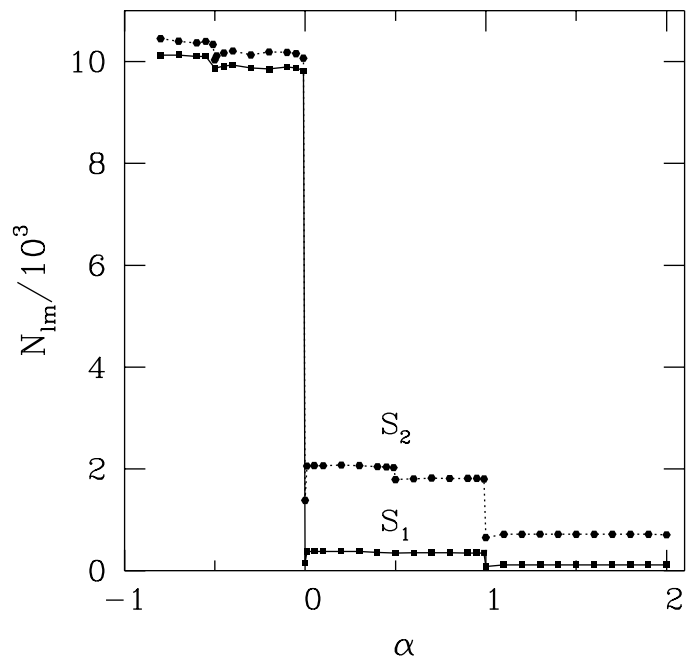

FIG. 6. Dependence of $N_{l m}$ on $\alpha$ for $S_{1}$ and $S_{2}$.

It should be noted that in Camacho's model 9] $T_{f}$ was found to increase with $\alpha(\alpha<0)$ linearly. Our results presented in Figures 4 and 5 show that the region of $\alpha$ where one can observe the linear dependence is rather narrow. Such region becomes much wider, for example, in the case of the 27monomer chain in three dimensions (the results are not shown). Overall, the results for $T_{f}$, shown in Fig. 4 and 5 , demonstrate that the non-native contact repulsive energies improve both the thermodynamic stability and dynamical characteristics of folding.
We now focus on the $\alpha$ dependence of the temperature at which the folding time is minimal, $T_{\min }$. The results for $S_{1}$ and $S_{2}$ are shown in Fig. 4 and Fig. 5. For positive values of $\alpha, T_{f}$ and $T_{\min }$ are comparable for both sequences and they should be good folders [13]. For large negative values of $\alpha$, $T_{\text {min }}$ is bigger than $T_{f}$ but $S_{1}$ and $S_{2}$ remain good folders by the Thirumalai-Camacho criterion [17] 18] - the peaks of the structural susceptibility and the specific heat coincide for this interval of $\alpha$. (For $\alpha=0$, the coincidence of the peaks is, in fact, a general feature of the Go models because the proximity to the native state and occurence of rapid changes in an average energy as a function of $T$ are both controlled by establishment of the same native contacts).

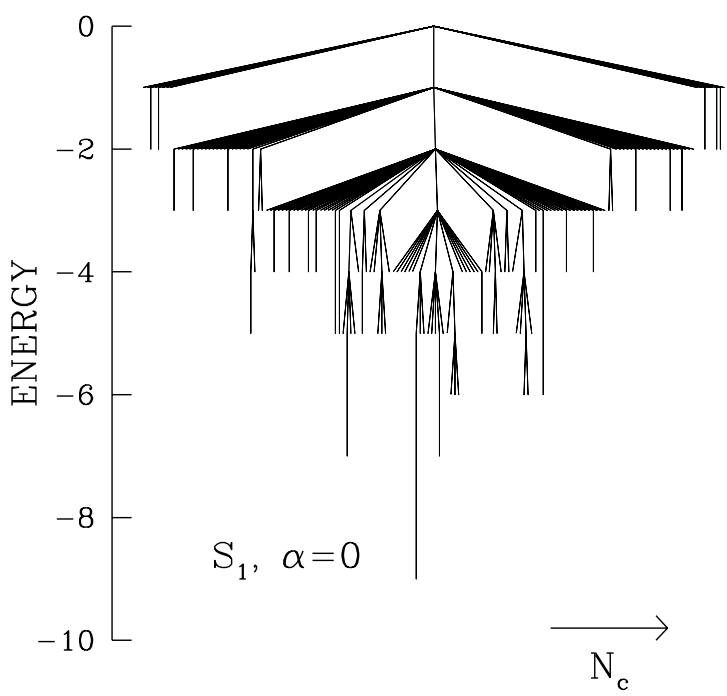

FIG. 7. The disconnectivity graph for $S_{1}$ and $\alpha=0$. $N_{c}$ is a symbolic notation for the index labelling the local energy minima.

Fig. 4 and 5 also show the dependence of the minimal folding time, $t_{\min }$, on $\alpha$. The dependence seems to saturate at large values of $\alpha$. If one extends the Camacho result to positive $\alpha$ then $t_{\min }$ should decrease with $\alpha$ exponentially [9]. Our results suggest that such conclusion is valid only for $\alpha<0$ but not for $\alpha>0$. Thus, the repulsive non-native contact energies make the polypeptide chain to fold faster which is similar to what has been observed in Ref. 此, 6.

In order to understand why the repulsion improves the folding so much we study the dependence of number of local minima, $N_{l m}$, on $\alpha$. The results for $S_{1}$ and $S_{2}$ are shown in Fig.6. $N_{l m}$ of $S_{1}$ is found to 
be smaller than for $S_{2}$. Clearly, the number of local minima strongly depends on $\alpha$ and for $\alpha>0$ it become by 2 orders of magnitude smaller than that for $\alpha<0$. So, the positive values of $\alpha$ make the energy landscape less rugged and the folding dynamics get faster.

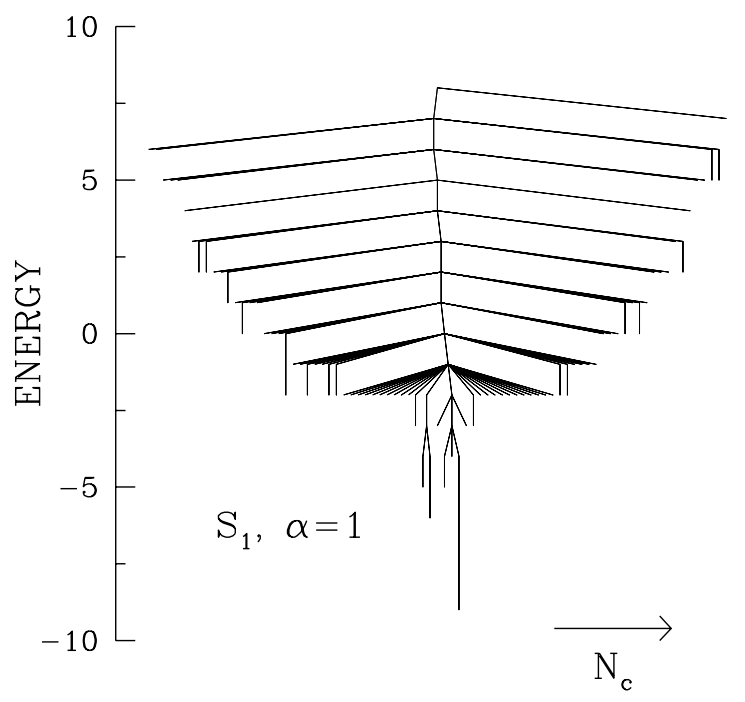

FIG. 8. The same as in Fig. 7 but for $\alpha=1$.

In order to get more insight into the nature of the energy landscapes in the models studied here, we use the disconnectivity graph technique which maps the potential energy surface onto the set of local minima [8, 19]. The technique involves checking what local energy minima are connected by pathways (sets of moves that are allowed kinetically) that do not exceed a given total threshold energy. For each value of this energy the minima are divided into disconnected sets of mutually accessible minima separated by barriers. The local minima which share the lowest energy threshold are joined at a node by lines and are called a basin corresponding to the threshold. The procedure of construction is stoped when one gets only one basin for all of the minima. For $S_{1}$ the number of local minima is equal to 152 and 81 for $\alpha=0$ and $\alpha=1$, respectively. The construction of the graphs, therefore, may be done exactly. The disconnectivity graphs obtained for $S_{1}$ and $\alpha=0$ and $\alpha=1$ are shown in Fig. 7 and Fig. 8, respectively. For both values of $\alpha$ sequence $S_{1}$ is a good folder and consequently, the structure corresponding to a folding funnel is clearly visible. However, the funnel for the repulsive case of $\alpha=1$ has a significantly less complex pattern than for $\alpha=0$. Moreover, the repulsive non-native interactions reduce the number of the local energy minima that have links to the native state below any predetermined energy threshold. Thus, the comparison of the disconnectivity graphs also shows that the repulsion may facilitate the folding substantially.

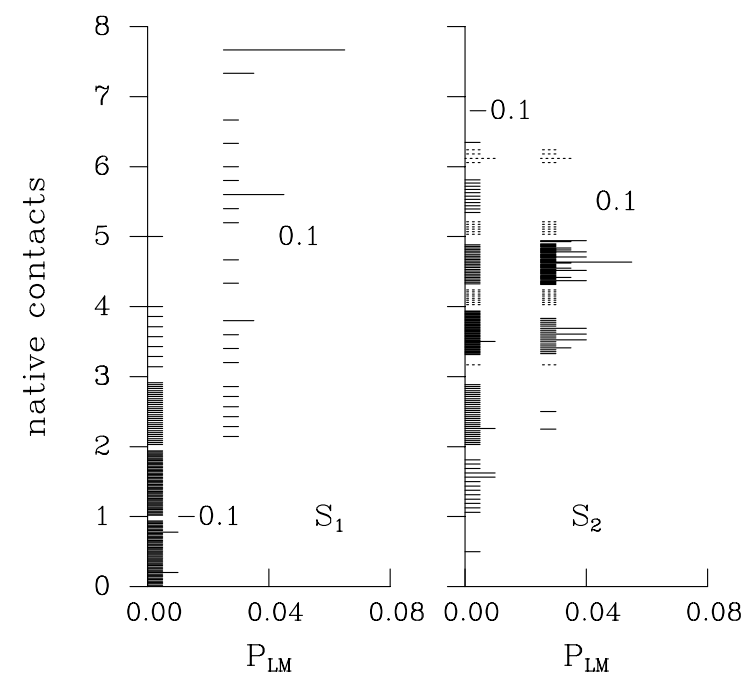

FIG. 9. The density of the ( $V$-shape) local minima, $P_{L M}$, in which the system gets trapped at $T=0$ is plotted versus the number of native contacts for $S_{1}$ and $S_{2}$. We choose $\alpha=0.1$ and $\alpha=-0.1$. The results are obtained for the batch of 200 trajectories. In the case of $S_{1}$ the local minima are different for $\alpha=-0.1$ and $\alpha=0.1$. For $S_{2}$ there are 19 common local minima which are marked by dotted lines.

We now address the question of what happens with trapped local minima when $\alpha$ changes from negative to positive values. Fig. 9 shows the histogram of the local minima for $\alpha=-0.1$ and 0.1 for sequence $S_{1}$ and $S_{2}$. Interestingly, for $S_{1}$ none of the local minima obtained for $\alpha=-0.1$ appears for $\alpha=0.1$. The situation changes dramatically for $S_{2}$ for which 19 local minima are common for both $\alpha=-0.1$ and $\alpha=0.1$. Our results suggest that for the sequence with $\delta E=0$ the local minima in which the chain is trapped at the negative values of $\alpha$ are effectively avoided if $\alpha$ is changed to positive values.

In conclusion, we state that for the simple extended Go model $T_{f}\left(t_{\min }\right)$ increases (decreases) with the non-native contact energy and it gets saturated for large values of $\alpha$. The complexity of the disconnectivity trees becomes reduced on making $\alpha$ more and more repulsive so that some two dimensional sequences may even lose the Arrhenius like behavior of $t_{\text {fold }}$ at low temperatures. It would 
be interesting to determine whether there are any three-dimensional Go-like sequences that fold even at $T=0$. Another important question is what kind of an effective parameter $\alpha$, or its conceptual equivalent, characterizes real proteins.

We thank Jayanth R. Banavar, P. Garstecki and T. X. Hoang for many useful discussions. This work was supported by KBN (grant No. 2P03B-025-13).

[1] K. A. Dill, S. Bromberg, S. Yue, K. Fiebig, K. M. Yee, D. P. Thomas, and H. S. Chan, Protein Science 4, 561 (1995).

[2] J. D. Bryngelson, J. N. Onuchic, N. D. Socci, and P. G. Wolynes, Proteins 21, 167 (1995).

[3] N. Go and H. Abe, Biopolymers 20, 1013 (1981).

[4] I. Shrivastava, S. Vishveshwara, M. Cieplak , A. Maritan, and J. R. Banavar, Proc. Natl. Acad. Sci. USA 92, 9206 (1995).

[5] A. M. Gutin, V. I. Abkevich, and E. I. Shakhnovich, Biochemistry 34, 3066 (1995).

[6] H. S. Chan and K. A. Dill, Proteins: Struct. Funct. Genet. 33, 2 (1998).

[7] H. S. Chan and K. A. Dill, J. Chem. Phys. 100, 9238 (1994).

[8] O. M. Becker and M. Karplus, J. Chem. Phys. 106, 1495 (1997).

[9] C. J. Camacho, Phys. Rev. Lett. 77, 2324 (1996).

[10] A. Gutin, V. Abkevich, and E. Shakhnovich, Phys. Rev. Lett. 80, 208 (1998).

[11] M. Cieplak, M. Henkel, J. Karbowski, and J. R. Banavar, Phys. Rev. Lett. 80, 3654 (1998); M. Cieplak, M. Henkel, and J. R. Banavar, J. Cond. Matt. (in press).

[12] H. S. Chan and K. A. Dill, J. Chem. Phys. 99, 2116 (1994).

[13] N. D. Socci, J. N. Onuchic, J. Chem. Phys. 101, 1519 (1994); M. Cieplak and J.R. Banavar, Folding and Design 2, 235 (1997)

[14] A. M. Gutin, V. I. Abkevich, and E. I. Shakhnovich, Phys. Rev. Lett. 77, 5433 (1996)

[15] K. F. Lau and K. A. Dill, Macromolecules 22, 3986 (1989)

[16] A. Dinner, A. Sali, M. Karplus, and E. Shakhnovich, J. Chem. Phys. 101, 1444 (1994)

[17] D. Thirumalai, J. Phys. I (France) 5, 1457 (1995)

[18] C. J. Camacho and D. Thirumalai, Proc. Natl. Acad. Sci. USA 90, 6369 (1993).

[19] D. J. Wales, M. A. Miller, and T. R. Walsh, Nature 394, 758 (1998). 